\section{Schwereloses Wissen}

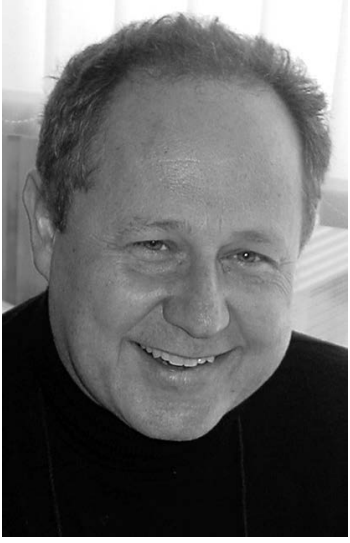

Prof. Dr. med. Manfred Wildner

Liebe Leserinnen und Leser,

das vorliegende Heft enthält neben den ausgewählten Beiträgen in gedruckter Form einen zunächst unsichtbaren Mehrwert: ein rein elektronisches Open-access-Schwerpunktheft (Supplement). Während Sie die Druckbeiträge wie gewohnt durchblättern und durchlesen können, sind die Open-accessBeiträge mit dem Schwerpunkt Versorgungsforschung nur im Internet zugänglich. Hier allerdings zum freien Download für alle. Diese Beiträge sind wie die gedruckten Beiträge in den Literaturdatenbanken wie z.B. Pubmed verzeichnet und können auch von dort aus gefunden werden.

Wir hoffen, dass diese „crossmediale Premiere“ für Sie in dem einen oder anderen Beitrag, vielleicht auch mit dem dortigen Editorial, auch in dieser Schwerelosigkeit einen Mehrwert bieten kann.

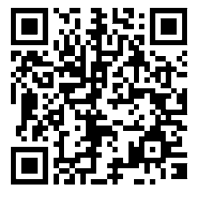

Schauen Sie gleich mal rein: thieme.de/versorgungsforschung
Supplement

Versorgungsforschung

\section{Panorama}

e83 Gesundheitsförderung - Zehn Herausforderungen im öffentlichen Gesundheitswesen

e84 Patientenversorgung - Aktuelle Trends in der Telemedizin

e85 Medizin weltweit - Medizinische Versorgung von Flüchtlingen

\section{Editorial}

e87 Die Macht des Wortes M. Wildner

\section{Originalarbeiten}

e89 Wie bewerten chronisch Kranke die organisatorischen und interpersonellen Aspekte ihrer haus- und fachärztlichen Versorgung im Vergleich? Ergebnisse einer deutschlandweiten Befragung J. Röttger, R. Busse

e97 Pflegerische Versorgungsdefizite in deutschen Krankenhäusern - Ergebnisse einer bundesweiten Befragung von Krankenhaus-Leitungspersonen A. Reifferscheid, N. Pomorin, J. Wasem

e103 Umsetzung und Nutzenbewertung von Handlungsempfehlungen zur Patientensicherheit in der ambulanten operativen Versorgung C. Monaca, M. Buchmann, T. Manser

e110 Anforderungen und Anwendungshinweise für den Einsatz von Qualitätsindikatoren in der medizinischen Versorgung: Ergebnisse eines systematischen Reviews S. Rode, V. Ries, T. Petzold, U. Buch, F. Untersweg, B. Fischer

e120 Welche Rückenschmerzpatienten profitieren von einer multimodalen Schmerztherapie? Eine komparative Kostenanalyse auf Basis von GKV-Routinedaten S. Behrendt, H. Kulas, U. Marschall, M. Steffens, G. Schiffhorst, H.-H. Bleß

e128 Überforderte pflegende Angehörige bei der Versorgung am Lebensende - was bieten Hausarztpraxen zur Entlastung an? K. Krug, R. Bölter, R. A. Ballhausen, P. Engeser, F. Peters-Klimm

e135 Eignung und Anwendung von GKV-Routinedaten zur Überprüfung von Versorgungsleitlinien am Beispiel der Indikation Linksherzinsuffizienz S. Neubauer, J. Zeidler, T. Schilling, S. Engel, R. Linder, F. Verheyen, A. Haverich, J.-M. G. von der Schulenburg

\section{Konsensstatement}

e145 STandardisierte BerichtsROutine für Sekundärdaten Analysen (STROSA) - ein konsentierter Berichtsstandard für Deutschland, Version 2 E. Swart, E. M. Bitzer, H. Gothe, M. Harling, F. Hoffmann, D. Horenkamp-Sonntag, B. Maier, S. March, T. Petzold, R. Röhrig, A. Rommel, T. Schink, C. Wagner, S. Wobbe, J. Schmitt 